\title{
Correction to: Do Testosterone and Cortisol Jointly Relate to Adolescent Dominance? A Pre-registered Multi-method Interrogation of the Dual-Hormone Hypothesis
}

\author{
Allison N. Shields ${ }^{1} \cdot$ Cassandra M. Brandes $^{1} \cdot$ Kathleen W. Reardon $^{2}$. \\ Raul A. España ${ }^{1} \cdot$ Jennifer L. Tackett ${ }^{1}$ \\ Published online: 19 August 2021 \\ (c) Springer Nature Switzerland AG 2021
}

\section{Correction to: Adaptive Human Behavior and Physiology (2021) 7:183-208 https://doi.org/10.1007/s40750-021-00167-3}

The original published version of this article contained mistakes. There were errors in Table 2, Supplementary Tables 5 and 6, and the Results.

In Table 2, values in the " $\mathrm{SE}_{\beta}$ " and " $\mathrm{CI}_{\beta}$ " columns were incorrect. Values in the " $\beta$ " column for all T x C and T x C x Gender interaction terms were also incorrect. The correct values are below in a corrected version of Table 2.

All confidence intervals and beta values for $\mathrm{T} \times \mathrm{C}$ interactions were incorrect in Supplementary Table 5. Beta values for $\mathrm{T} \times \mathrm{C}$ and $\mathrm{T} \times \mathrm{C}$ x Gender interactions were incorrect in Supplementary Table 6. The corrected supplementary tables can be found at https://osf.io/kzne2/.

Paragraphs five through twelve of the "Results" section, "Dual-Hormone Hypothesis," reflect these errors. The Results section should read as follows:

Observer-Rated Dominance

For salivary hormones, there was a small main effect of $\mathrm{T}$ on observer-rated dominance, such that adolescents with lower levels of $\mathrm{T}$ were rated as more dominant, on average $(\beta=-0.13,95 \%$ CI $[-0.24,-0.02], p=0.02)$. Main effects of $\mathrm{C}(\beta=0.07$,

The original article can be found online at https://doi.org/10.1007/s40750-021-00167-3.

Jennifer L. Tackett

jennifer.tackett@northwestern.edu

1 Department of Psychology, Northwestern University, 2029 Sheridan Rd., Evanston, IL 60208, USA

2 Center for Applied Psychological and Family Studies, The Family Institute at Northwestern University, 618 Library Pl, Evanston, IL 60201, USA 
Table 2 Hierarchical Regression Results Predicting Dominance

\begin{tabular}{|c|c|c|c|c|c|c|c|c|}
\hline Step & Variable & $\mathrm{B}$ & $\beta$ & $\mathrm{SE}_{\beta}$ & $\mathrm{CI}_{\beta}$ & $p$ & $\mathrm{R}^{2}$ & $\mathrm{~F}(p)$ \\
\hline & OV: Observer-rater Dor & ninance & & & & & & \\
\hline & Model 1: Salivary Horn & hones & & & & & & \\
\hline \multirow[t]{2}{*}{1} & Saliva time & $<0.01$ & 0.02 & 0.05 & {$[-0.07,0.12]$} & 0.64 & 0.27 & $54.73(<0.001)$ \\
\hline & Leader-Follower Time & -0.59 & -0.52 & 0.05 & {$[-0.61,-0.42]$} & $<0.001$ & & \\
\hline \multirow[t]{3}{*}{2} & Gender & $<0.01$ & $<0.01$ & 0.05 & {$[-0.11,0.11]$} & 0.998 & 0.30 & $21.13(<0.001)$ \\
\hline & $\mathrm{T}$ & -0.12 & -0.13 & 0.06 & {$[-0.24,-0.02]$} & 0.02 & & \\
\hline & $\mathrm{C}$ & 0.10 & 0.07 & 0.06 & {$[-0.05,0.18]$} & 0.24 & & \\
\hline 3 & $\mathrm{~T} \times \mathrm{C}$ & -0.05 & -0.03 & 0.06 & {$[-0.14,0.08]$} & 0.55 & 0.30 & $17.62(<0.001)$ \\
\hline \multirow[t]{2}{*}{4} & T x C x Gender & 0.31 & 0.10 & 0.06 & {$[-0.01,0.22]$} & 0.07 & 0.32 & $12.53(<0.001)$ \\
\hline & Model 2: Hair Hormon & & & & & & & \\
\hline 1 & Leader-Follower Time & -0.58 & -0.51 & 0.05 & {$[-0.61,-0.42]$} & $<0.001$ & 0.26 & $110.80(<0.001)$ \\
\hline \multirow[t]{3}{*}{2} & Gender & 0.04 & 0.02 & 0.06 & {$[-0.10,0.14]$} & 0.74 & 0.29 & $20.00(<0.001)$ \\
\hline & $\mathrm{T}$ & 0.06 & 0.07 & 0.06 & {$[-0.05,0.19]$} & 0.27 & & \\
\hline & $\mathrm{C}$ & -0.02 & -0.03 & 0.06 & {$[-0.15,0.10]$} & 0.65 & & \\
\hline 3 & $\mathrm{~T} \times \mathrm{C}$ & -0.06 & -0.09 & 0.06 & {$[-0.21,0.03]$} & 0.14 & 0.29 & $16.53(<0.001)$ \\
\hline \multirow[t]{3}{*}{4} & T x C x Gender & -0.12 & -0.08 & 0.06 & {$[-0.20,+0.04]$} & 0.20 & 0.31 & $10.82(<0.001)$ \\
\hline & OV: AMS Dominance-1 & Parent & & & & & & \\
\hline & Model 3: Salivary Horn & hones & & & & & & \\
\hline \multirow[t]{4}{*}{1} & Saliva time & $<0.01$ & -0.02 & 0.06 & {$[-0.14,0.10]$} & 0.72 & 0.03 & $1.73(0.14)$ \\
\hline & Digits Forward & -0.01 & -0.05 & 0.07 & {$[-0.20,0.09]$} & 0.45 & & \\
\hline & Digits Backward & $<0.01$ & 0.01 & 0.07 & {$[-0.14,0.15]$} & 0.93 & & \\
\hline & Trails B Time & -0.002 & -0.16 & 0.07 & {$[-0.29,-0.03]$} & 0.01 & & \\
\hline \multirow[t]{3}{*}{2} & Gender & 0.04 & 0.06 & 0.07 & {$[-0.08,0.20]$} & 0.38 & 0.03 & $1.00(0.43)$ \\
\hline & $\mathrm{T}$ & -0.01 & -0.05 & 0.07 & {$[-0.19,0.10]$} & 0.54 & & \\
\hline & $\mathrm{C}$ & 0.01 & 0.02 & 0.07 & {$[-0.13,0.17]$} & 0.79 & & \\
\hline 3 & $\mathrm{~T} \times \mathrm{C}$ & $<0.01$ & 0.01 & 0.07 & {$[-0.13,0.15]$} & 0.89 & 0.03 & $0.88(0.54)$ \\
\hline \multirow[t]{2}{*}{4} & T x C x Gender & 0.01 & 0.01 & 0.07 & {$[-0.14,0.15]$} & 0.90 & 0.04 & $0.72(0.72)$ \\
\hline & Model 4: Hair Hormon & & & & & & & \\
\hline \multirow[t]{3}{*}{1} & Digits Forward & -0.01 & -0.04 & 0.07 & {$[-0.18,0.10]$} & 0.56 & 0.02 & $2.10(0.10)$ \\
\hline & Digits Backward & $<0.01$ & $<0.01$ & 0.07 & {$[-0.14,0.15]$} & 0.95 & & \\
\hline & Trails B Time & -0.001 & -0.16 & 0.06 & {$[-0.28,-0.03]$} & 0.02 & & \\
\hline \multirow[t]{3}{*}{2} & Gender & 0.05 & 0.07 & 0.08 & {$[-0.08,0.22]$} & 0.37 & 0.03 & $0.85(0.53)$ \\
\hline & $\mathrm{T}$ & 0.01 & 0.03 & 0.08 & {$[-0.12,0.19]$} & 0.66 & & \\
\hline & $\mathrm{C}$ & 0.03 & 0.11 & 0.08 & {$[-0.04,0.27]$} & 0.15 & & \\
\hline 3 & $\mathrm{~T} \times \mathrm{C}$ & $<0.01$ & $<0.01$ & 0.07 & {$[-0.15,0.15]$} & 0.998 & 0.03 & $0.73(0.65)$ \\
\hline \multirow[t]{3}{*}{4} & T x C x Gender & -0.05 & -0.09 & 0.07 & {$[-0.24,0.06]$} & 0.23 & 0.05 & $0.88(0.55)$ \\
\hline & OV: AMS Dominance- & Youth & & & & & & \\
\hline & Model 5: Salivary Horn & nones & & & & & & \\
\hline \multirow[t]{4}{*}{1} & Saliva Time & $<0.01$ & 0.13 & 0.06 & {$[0.02,0.25]$} & 0.02 & 0.07 & $5.62(<0.001)$ \\
\hline & Digits Forward & $<0.01$ & 0.03 & 0.07 & {$[-0.10,0.17]$} & 0.61 & & \\
\hline & Digits Backward & $<0.01$ & $<0.01$ & 0.07 & {$[-0.13,0.14]$} & 0.95 & & \\
\hline & Trails B Time & -0.002 & -0.23 & 0.06 & {$[-0.35,-0.11]$} & $<0.001$ & & \\
\hline
\end{tabular}


Table 2 (continued)

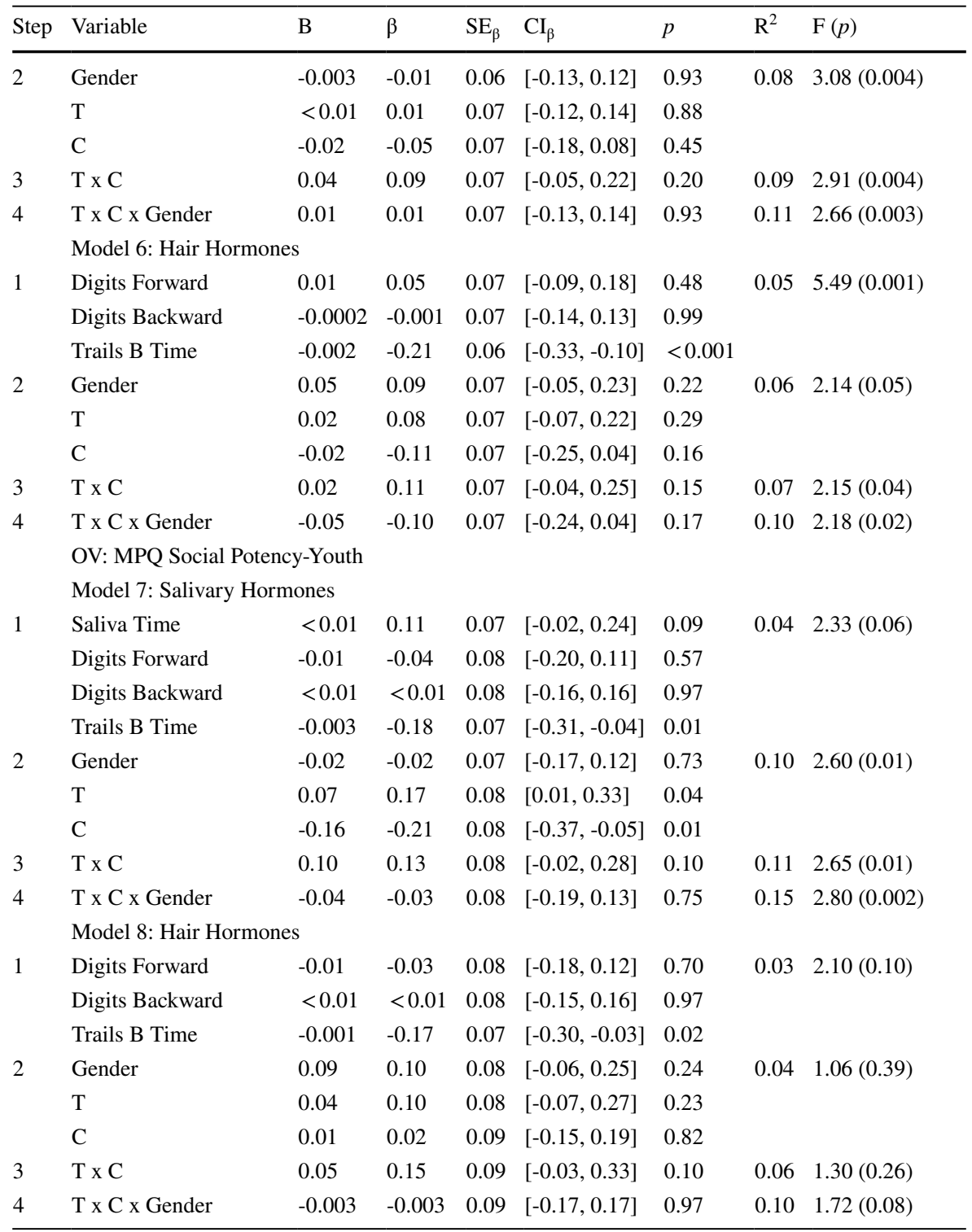

Note. In Step 3, models included all main effects and in Step 4, models included all main effects and lower-order two-way interactions. Full model results can be found on the OSF page for this project (https://osf.io/9n8gf/). SE, Standard Error; CI, Confidence Interval; OV, Outcome Variable; T, Testosterone; C, Cortisol; AMS, Achievement Motivation Scale; MPQ, Multidimensional Personality Questionnaire

95\% CI $[-0.05,0.18], p=0.24)$ and gender $(\beta<0.01,95 \%$ CI $[-0.11,0.11], p>0.99)$ on observer-rated dominance were nonsignificant. The $\mathrm{T} \times \mathrm{C}$ interaction was nonsignificant $\left(\beta=-0.03,95 \%\right.$ CI $[-0.14,0.08], p=0.55, \Delta \mathrm{R}^{2}<0.01$; Figure 1a). The 
$\mathrm{T} \times \mathrm{C} \times$ gender interaction was also nonsignificant $(\beta=0.10,95 \% \mathrm{CI}[-0.01,0.22]$, $\left.p=0.07, \Delta \mathrm{R}^{2}=0.02\right)$. However, in models run separately by participant gender, the direction of the $\mathrm{T} \times \mathrm{C}$ interaction was negative in males $(\beta=-0.12,95 \%$ CI $[-0.30$, $+0.06], p=0.17)$ and positive in females $(\beta=0.08,95 \%$ CI [-0.06, 0.22], $p=0.27)$.

For hair hormones, main effects of T $(\beta=0.07,95 \%$ CI $[-0.05,0.19], p=0.27)$, C $(\beta=-0.03,95 \%$ CI $[-0.15,0.10], p=0.65)$, and gender $(\beta=0.02,95 \%$ CI $[-0.10$, $0.14], p=0.74)$ on observer-rated dominance were nonsignificant. The $\mathrm{T} \times \mathrm{C}$ interaction was nonsignificant $\left(\beta=-0.09,95 \%\right.$ CI [-0.21, 0.03], $p=0.14, \Delta \mathrm{R}^{2}<0.01$; Figure 2a). The $\mathrm{T} \times \mathrm{C} \times$ gender interaction was also nonsignificant $(\beta=-0.08,95 \% \mathrm{CI}$ $\left.[-0.20,+0.04], p=0.20, \Delta \mathrm{R}^{2}=0.01\right)$, though the $\mathrm{T} \times \mathrm{C}$ interaction was positive in males $(\beta=0.0495 \%$ CI $[-0.17,0.25], p=0.69)$ and negative in females $(\beta=-0.13$, $95 \%$ CI [-0.29, 0.03], $p=0.10)$.

\section{Parent-Reported AMS Dominance}

For salivary hormones, main effects of $\mathrm{T}(\beta=-0.05,95 \% \mathrm{CI}[-0.19,0.10]$, $p=0.54), \mathrm{C}(\beta=0.02,95 \% \mathrm{CI}[-0.13,0.17], p=0.79)$, and gender $(\beta=0.06,95 \% \mathrm{CI}$ $[-0.08,0.20], p=0.38)$ on parent-reported dominance were nonsignificant. The $\mathrm{T} \times \mathrm{C}$ interaction was nonsignificant $\left(\beta=0.01,95 \% \mathrm{CI}[-0.13,0.15], p=0.89, \Delta \mathrm{R}^{2}<0.01\right.$; Figure 1b) and the direction of this effect was equivalent in males and females. The $\mathrm{T} \times \mathrm{C} \times$ gender interaction was also nonsignificant $(\beta=0.01,95 \%$ CI $[-0.14,0.15]$, $\left.p=0.90, \Delta \mathrm{R}^{2}<0.01\right)$.

For hair hormones, main effects of $\mathrm{T}(\beta=0.03,95 \%$ CI $[-0.12,0.19], p=0.66)$, $\mathrm{C}(\beta=0.11,95 \% \mathrm{CI}[-0.04,0.27], p=0.15)$, and gender $(\beta=0.07,95 \%$ CI $[-0.08$, $0.22], p=0.37$ ) on parent-reported dominance were also nonsignificant. The $\mathrm{T} \times \mathrm{C}$ interaction was nonsignificant $\left(\beta<0.01,95 \%\right.$ CI $[-0.15,0.15], p>0.99, \Delta \mathrm{R}^{2}<0.01$; Figure 2b). The $\mathrm{T} \times \mathrm{C} \times$ gender interaction was also nonsignificant $(\beta=-0.09,95 \%$ CI $\left.[-0.24,0.06], p=0.23, \Delta \mathrm{R}^{2}=0.02\right)$, though the $\mathrm{T} \times \mathrm{C}$ interaction was positive in males $(\beta=0.13,95 \%$ CI $[-0.15,0.40], p=0.36)$ and negative in females $(\beta=-0.04$, $95 \%$ CI $[-0.24,0.16], p=0.69)$.

\section{Youth-Reported AMS Dominance}

For salivary hormones, main effects of $\mathrm{T}(\beta=0.01,95 \% \mathrm{CI}[-0.12,0.14]$, $p=0.88), \mathrm{C}(\beta=-0.05,95 \% \mathrm{CI}[-0.18,0.08], p=0.45)$, and gender $(\beta=-0.01,95 \%$ CI $[-0.13,0.12], p=0.93)$ on youth-reported dominance were nonsignificant. The $\mathrm{T} \times \mathrm{C}$ interaction was nonsignificant $(\beta=0.09,95 \% \mathrm{CI}[-0.05,0.22], p=0.20$, $\Delta \mathrm{R}^{2}=0.01$; Figure 1c) and the direction of this effect was equivalent in males and females. The $\mathrm{T} \times \mathrm{C} \times$ gender interaction was also nonsignificant $(\beta=0.01,95 \% \mathrm{CI}$ $\left.[-0.13,0.14], p=0.93, \Delta \mathrm{R}^{2}=0.02\right)$.

For hair hormones, main effects of $\mathrm{T}(\beta=0.08,95 \% \mathrm{CI}[-0.07,0.22], p=0.29)$, C $(\beta=-0.11,95 \%$ CI $[-0.25,0.04], p=0.16)$, and gender $(\beta=0.09,95 \%$ CI $[-0.05$, $0.23], p=0.22)$ on youth-reported dominance were also nonsignificant. The $\mathrm{T} \times \mathrm{C}$ interaction was nonsignificant $\left(\beta=0.11,95 \% \mathrm{CI}[-0.04,0.25], p=0.15, \Delta \mathrm{R}^{2}=0.01\right.$; Figure 2c) and the direction of this effect was equivalent in males and females. The $\mathrm{T} \times \mathrm{C} \times$ gender interaction was also nonsignificant $(\beta=-0.10,95 \% \mathrm{CI}[-0.24,0.04]$, $\left.p=0.17, \Delta \mathrm{R}^{2}=0.03\right)$. 


\section{Youth-Reported MPQ Social Potency}

For salivary hormones, there was a small main effect of $\mathrm{T}$ on youth-reported social potency, such that adolescents with higher levels of salivary $\mathrm{T}$ were higher in social potency, on average ( $\beta=0.17,95 \% \mathrm{CI}$ [0.01, 0.33], $p=0.04)$. There was also a small main effect of $\mathrm{C}$, such that adolescents with lower levels of salivary $\mathrm{C}$ were higher in social potency, on average $(\beta=-0.21,95 \%$ CI $[-0.37,-0.05], p=0.01)$. The main effect of gender on social potency was nonsignificant $(\beta=-0.02,95 \%$ CI $[-0.17,0.12], p=0.73)$. The $\mathrm{T} \times \mathrm{C}$ interaction was nonsignificant $(\beta=0.13,95 \% \mathrm{CI}$ $[-0.02,0.28], p=0.10, \Delta \mathrm{R}^{2}=0.01$; Figure 1d) and the direction of this effect was equivalent in males and females. The $\mathrm{T} \times \mathrm{C} \times$ gender interaction was also nonsignificant $\left(\beta=-0.03,95 \%\right.$ CI $\left.[-0.19,0.13], p=0.75, \Delta \mathrm{R}^{2}=0.04\right)$.

For hair hormones, main effects of $\mathrm{T}(\beta=0.10,95 \% \mathrm{CI}[-0.07,0.27], p=0.23), \mathrm{C}$ $(\beta=0.02,95 \%$ CI $[-0.15,0.19], p=0.82)$ and gender $(\beta=0.10,95 \%$ CI $[-0.06,0.25]$, $p=0.24)$ on youth-reported social potency were nonsignificant. The $\mathrm{T} \times \mathrm{C}$ interaction was nonsignificant $\left(\beta=0.15,95 \%\right.$ CI [-0.03, 0.33], $p=0.11, \Delta \mathbf{R}^{2}=0.02$; Figure 2d) and the direction of this effect was equivalent in males and females. The $\mathrm{T} \times \mathrm{C} \times$ gender interaction was also nonsignificant $(<-0.01<\beta<0,95 \%$ CI $[-0.17$, $0.17], p=0.97, \Delta \mathrm{R}^{2}=0.05$ ).

Supplementary Information The online version contains supplementary material available at https://doi. org/10.1007/s40750-021-00171-7.

Publisher's Note Springer Nature remains neutral with regard to jurisdictional claims in published maps and institutional affiliations. 